\title{
The $\frac{1}{\mathrm{r}}$ Expansion for the Critical Multiple Well Problem
}

\author{
Raphael Høegh-Krohn* and Mohammed Mebkhout** \\ Centre de Physique Théorique, C.N.R.S. Luminy, Case 907, F-13288 Marseille Cedex 9, France
}

\begin{abstract}
We consider the critical multiple well problem

$$
H=-\Delta+\sum_{i=1}^{n} V\left(x-r x_{i}\right)
$$

where $-\Delta+V(x)$ has a zero energy resonance. We prove that all eigenvalues and resonances of $H$ tending to zero as $1 / r^{2}$ are analytic in $1 / r$. We give an explicit equation for the lowest nonvanishing coefficient in the $1 / r$ expansion for any of these eigenvalues or resonances and observe that $H$ has infinitely many resonances tending to zero. For $n=2$ and $n=3$, we compute the coefficients explicitly and for $n=2$, we also give the next coefficient in the $1 / r$ expansion.
\end{abstract}

\section{Introduction}

In this paper, we study the critical multiple well problem, i.e. the asymptotic behavior of the eigenfunctions and resonances of

$$
H_{r}=-\Delta+\sum_{i=1}^{n} V\left(x-r x_{i}\right)
$$

in $L_{2}\left(R^{3}\right)$ as $r \rightarrow \infty$, where $V$ is a potential of compact support such that $-\Delta+V$ has a zero energy resonance. We find that there are infinitely many resonances and finitely many eigenvalues, which tend to zero as $r \rightarrow \infty$. For these resonances and eigenvalues we prove that they are analytic in $1 / r$ and we give the corresponding $1 / r$ expansion. The eigenvalue tending to zero for $n=2$ was studied by Klaus and Simon in [1] where they proved that this eigenvalue behaved like $E_{0}(r)$ $=-\sigma_{0}^{2} r^{-2}+O\left(r^{-3}\right)$, where $\sigma_{0}$ is the unique real solution of $\sigma=e^{-\sigma}$. We extend

* Université de Provence, Centre Saint Charles, and Centre de Physique Théorique, C.N.R.S. Marseille

** Université d'Aix-Marseille II, Faculté des Sciences de Luminy and Centre de Physique Théorique, Section 2, C.N.R.S. Marseille 
their result by showing that the eigenvalue or resonance tending to zero as fast as $r^{-2}$ are all analytic in $1 / r$, and they are given by

$$
E_{n}(r)=-\sigma_{n}^{2} r^{-2}+b \cdot \sigma_{n}^{3}\left(1-\sigma_{n}\right)^{-1} r^{-3}+O\left(r^{-4}\right),
$$

where $\sigma_{n}$ are all the complex solutions of $\sigma= \pm e^{-\sigma}$ and $b$ is a constant that depends on the potential $V$. One of the interesting features of this expansion is that the coefficients to the lowest order are universal and do not depend on the potential at all. To the next order the coefficient does depend on the potential but only through a constant that is the same for all the resonances and is given by

$$
b=\left(V, \psi_{0}\right)^{-2}\left(\psi_{0}, V \psi_{0}\right)
$$

where $\psi_{0}$ is the zero energy resonance function, i.e. $(-\Delta+V) \psi_{0}=0$. We obtain similar results for all $n$. The noncritical double well was studied by Klaus in [2].

Our results in this note are a direct consequence of the short range expansion in [3]. The Hamiltonian in the short range expansion is given by

$$
H(\varepsilon)=-\Delta+\frac{1}{\varepsilon^{2}} \sum_{i=1}^{n} V\left(\frac{1}{\varepsilon}\left(x-x_{i}\right)\right)
$$

in $L_{2}\left(\mathbb{R}^{3}\right)$, where $V$ is a potential of compact support. In [3] it is proved that the eigenvalues and resonances of $H(\varepsilon)$ that remains bounded as $\varepsilon \rightarrow 0$ are all analytic in $\varepsilon$ and their expansion is given. Let now $U_{r}$ be the unitary scaling in $L_{2}\left(\mathbb{R}^{3}\right)$ given by $\left(U_{r} \psi\right)(x)=r^{3 / 2} \psi(r x)$. Then

$$
U_{r} H_{r} U_{r}^{-1}=r^{-2}\left[-\Delta+r^{2} \sum_{i=1}^{n} V\left(r\left(x-x_{i}\right)\right)\right],
$$

i.e.

$$
H\left(\frac{1}{r}\right)=U_{r} H_{r} U_{r}^{-1}
$$

Hence all the technical results needed are contained in [3].

\section{Resonances and Eigenvalues}

Let us consider the Schrödinger operator

$$
H=-\Delta+V
$$

where $\Delta$ is the Laplacian in $L_{2}\left(\mathbb{R}^{3}\right)$ and $V$ is a bounded measurable function on $\mathbb{R}^{3}$ with compact support. With $u=|V|^{1 / 2}$ and $v=(\operatorname{sign} V) u$, we easily see that the resolvent kernel of $H$ satisfies the following equation for $\operatorname{Im} k>0$

$$
\left(H-k^{2}\right)^{-1}(x, y)=G_{k}(x-y)-\left[G_{k} v\left(1+u G_{k} v\right)^{-1} u G_{k}\right](x, y),
$$

where $G_{k}=\left(-\Delta-k^{2}\right)^{-1}$, so that

$$
G_{k}(x-y)=(4 \pi|x-y|)^{-1} e^{i k|x-y|} .
$$


Hence

$$
\left(u G_{k} v\right)(x, y)=u(x) G_{k}(x-y) v(y)
$$

is a Hilbert-Schmidt kernel for all complex $k$ and thus $k \rightarrow\left(1+u G_{k} v\right)^{-1}$ is a meromorphic Hilbert-Schmidt valued function in the whole complex plane with poles at the points where -1 is an eigenvalue of the compact operator $u G_{k} v$ with kernel defined by (2.4). Thus for $x \neq y$ the resolvent kernel (2.2) is a meromorphic function of $k$ in the whole complex plane with poles independent of $x$ and $y$, which are those values of $k$ for which

$$
\varphi+u G_{k} v \varphi=0
$$

has a nontrivial solution, i.e. where -1 is an eigenvalue of the compact operator $u G_{v} v$.

The poles in the upper half-plane $\operatorname{Im} k>0$, the so-called physical half-plane, correspond of course to the eigenvalues of $H$ with values $E=k_{0}^{2}$, where $k_{0}$ is the position of the pole, since for $\operatorname{Im} k>0,(2.2)$ is the resolvent kernel.

The poles in the lower half-plane $\operatorname{Im} k<0$, the so-called unphysical half-plane are called resonances and do not correspond to eigenvalues for $H$.

Let $k_{0}$ be a pole of (2.2) so that (2.5) has a nontrivial solution $\varphi_{0}$ for $k=k_{0}$. Multiplying (2.5) by $v$ we get

$$
v \varphi_{0}+V G_{k} v \varphi_{0}=0
$$

and with $\psi_{0}=G_{k_{0}} v \varphi_{0}$ we have

$$
\left(-\Delta+V-k_{0}^{2}\right) \psi_{0}=0 .
$$

Hence in the physical half-plane $\operatorname{Im} k>0$ we have that $\psi_{0}=G_{k_{0}} v \varphi_{0}$ is the corresponding eigenfunction. In the unphysical half-plane, if $k_{0}$ is a resonance we call $\psi_{0}=G_{k_{0}} v \varphi_{0}$ the resonance function. It solves the eigenvalue equation (2.7), but is not square integrable.

On the real axis we have that a pole $k_{0}$ corresponds to an eigenvalue if and only if $\psi_{0}=G_{k_{0}} v \varphi_{0}$ is square integrable. If $\psi_{0}$ is not square integrable, we say that $k_{0}$ is a resonance.

We say that $H=-\Delta+V$ has a zero energy resonance if

$$
\int|x-y|^{-2}|V(x) V(y)| d x d y<\infty \text { and } \varphi_{0}+u G_{0} v \varphi_{0}=0
$$

has a nontrivial solution $\varphi_{0}$ so that $G_{0} v \varphi_{0}$ is not square integrable.

\section{The Multiple Well Problem}

The Schrödinger operator for the multiple well problem is

$$
H_{\varepsilon}=-\Delta+\sum_{i=1}^{n} V\left(x-\frac{1}{\varepsilon} x_{i}\right)
$$

where $-\Delta$ is the Laplacian in $L_{2}\left(\mathbb{R}^{3}\right)$ and $V$ is a bounded measurable function of compact support. As in the previous section let $u=|V|^{1 / 2}$ and $v=u \operatorname{sign} V$, so that $V=u v$. Let $u_{i}(x)=u\left(x-\frac{1}{\varepsilon} x_{i}\right)$ and $v_{i}(x)=v\left(x-\frac{1}{\varepsilon} x_{i}\right)$. For $\operatorname{Re} k$ large negative we 
have from (2.2)

$$
\begin{aligned}
\left(H_{\varepsilon}-k^{2}\right)^{-1}= & G_{k}(x-y)-\sum_{i} G_{k} v_{i} u_{i} G_{k}+\sum_{i j} G_{k} v_{i} u_{i} G_{k} v_{j} u_{j} \\
& -\sum_{i j} \sum_{i_{1}} G_{k} v_{i} u_{i} G_{k} v_{i_{1}} u_{i_{1}} G_{k} v_{j} u_{j} G_{k}+\ldots, \\
\left(H_{2}-k^{2}\right)^{-1}= & G_{k}-\sum_{i j} G_{k} v_{i}\left[\delta_{i j}-u_{i} G_{k} v_{j}+\sum_{i_{1}} u_{i} G_{k} u_{i_{1}} G_{k} v_{j} \ldots\right] u_{j} G_{k},
\end{aligned}
$$

which gives us

$$
\left(H_{\varepsilon}-k^{2}\right)^{-1}=G_{k}-\sum_{i j} G_{k} v_{i}\left[1+u_{i} G_{k} v_{j}\right]_{i j}^{-1} u_{j} G_{k},
$$

where $u_{i} G_{k} v_{j}$ is to be considered as an operator on $L_{2}\left(\mathbb{R}^{3}\right) \otimes \mathbb{C}^{n}$ which maps

$$
\bar{f}:\left(f_{1} \ldots f_{n}\right) \in L_{2}\left(\mathbb{R}^{3}\right) \otimes \mathbb{C}^{n}
$$

into

$$
\sum_{j} u_{i}(x) \int G_{k}(x-y) v_{j}(y) f_{j}(y) d y .
$$

Hence the poles of the resolvent kernel $\left(H_{\varepsilon}-k^{2}\right)^{-1}$ are the poles of the meromorphic function $k \rightarrow\left[1+u_{i} G_{k} v_{j}\right]_{i j}^{-1}$ with values in Hilbert-Schmidt operators on $L_{2}\left(\mathbb{R}^{3}\right) \otimes \mathbb{C}^{n}$. The poles of this function are of course the points $k \in \mathbb{C}$ so that

$$
f_{i}+u_{i} \sum_{j} G_{k} v_{j} f_{j}=0
$$

has a nontrivial solution $\bar{f}=\left(f_{1}, f_{2} \ldots f_{n}\right)$ in $L_{2}\left(\mathbb{R}^{3}\right) \otimes \mathbb{C}^{n}$. Set now

$$
\varphi_{i}(x)=f_{i}\left(x+\frac{1}{\varepsilon} x_{i}\right) \text {. }
$$

Then (3.5) takes the form

$$
\varphi_{i}(x)+u(x) \sum_{j} \int G_{k}\left(x-y+\frac{1}{\varepsilon}\left(x_{i}-x_{j}\right)\right) v(y) \varphi_{j}(y) d y=0,
$$

where we have used that $f_{i}(x)=\varphi_{i}\left(x-\frac{1}{\varepsilon} x_{i}\right)$ and $u_{i}(x)=u\left(x-\frac{1}{\varepsilon} x_{i}\right)$, $v_{i}(x)=v\left(x-\frac{1}{\varepsilon} x_{i}\right)$. Taking the diagonal term in (3.6) outside the sum we may write

$$
\varphi_{i}(x)+u G_{k} v \varphi_{i}+\sum_{j \neq i} u(x) \int G_{k}\left(x-y+\frac{1}{\varepsilon}\left(x_{i}-x_{j}\right)\right) v(y) \varphi_{j}(y) d y=0 .
$$

Hence we have the following result, using that $G_{k}\left(\frac{1}{\varepsilon} x\right)=\varepsilon G_{k / \varepsilon}(x)$.

Theorem 3.1. The eigenvalues and the resonances for the multiple well problem

$$
H_{\varepsilon}=-\Delta+\sum_{i=1}^{n} V\left(x-\frac{1}{\varepsilon} x_{i}\right)
$$

are the square of the complex numbers $k$ for which the equation 


$$
\varphi_{i}+u G_{k} v \varphi_{i}+\varepsilon \sum_{j \neq 1}^{n} u(x) \int G_{k / \varepsilon}\left((x-y)+x_{i}-y_{j}\right) v(y) \varphi_{j}(y) d y=0
$$

has a nontrivial solution in $L_{2}\left(\mathbb{R}^{3}\right) \otimes \mathbb{C}^{n}$. The corresponding eigenfunction or resonance function is given by

$$
\psi(x)=\sum_{i=1}^{n} \int G_{k}\left(z-y-\frac{1}{\varepsilon} x_{i}\right) v(y) \varphi_{i}(y) d y .
$$

\section{The Critical Multiple Well Problem}

By the critical multiple well problem we understand the study of the eigenvalues and resonances of the multiple well Hamiltonian

$$
H_{2}=-\Delta+\sum_{i=1}^{n} V\left(x-\frac{1}{\varepsilon} x_{i}\right)
$$

where $x_{1}, \ldots, x_{n}$ are $n$ distinct points in $\mathbb{R}^{3}$. By Theorem 3.1 the eigenvalues and resonances are the squares of the complex numbers $k$ for which the equation

$$
\varphi_{i}+u G_{k} v \varphi_{i}+\varepsilon \sum_{j \neq i}^{n} u(x) \int G_{k / \varepsilon}\left(\varepsilon(x-y)+x_{i}-x_{j}\right) v(y) \varphi_{j}(y) d y
$$

has a nontrivial solution $\left(\varphi_{1}, \ldots, \varphi_{n}\right)$ in $L_{2}\left(\mathbb{R}^{\prime \prime}\right) \otimes \mathbb{C}^{n}$. The corresponding eigenfunction or resonant function is given by:

$$
\psi(x)=\sum_{i=1}^{n} G_{k_{0}}\left(x-y-\frac{1}{\varepsilon} x_{i}\right) v(y) \varphi_{i}(y) d y .
$$

Let now $k(\varepsilon)^{2}$ be an eigenvalue or a resonance of the multiple well problem (5.1) such that $|k(\varepsilon)| \leqq c \varepsilon$, and write

$$
k^{\prime}(\varepsilon)=\frac{1}{\varepsilon} k(\varepsilon)
$$

Then (4.2) takes the form

$$
\varphi_{i}+u G_{\varepsilon k^{\prime}} v \varphi_{i}+\varepsilon \sum_{j \neq 1}^{n} u(x) \int G_{k^{\prime}}\left(\varepsilon(x-y)+x_{i}-x_{j}\right) v(y) \varphi_{j}(y) d y=0 .
$$

This equation has been extensively studied in [3], where it was proved in Section [3] that (5.5) has solutions which are bounded as $\varepsilon \rightarrow 0$ only if

$$
\varphi_{0}+u G_{0} v \varphi_{0}=0
$$

has a nontrivial solution in $L^{2}\left(\mathbb{R}^{3}\right)$. According to Sect. 2 this is equivalent to $-\Delta+V$ having a zero energy resonance or eigenvalue. In [3] it is proved that

$$
\psi_{0}(x)=G_{0} v \varphi_{0}
$$

is in $L_{2}\left(\mathbb{R}^{3}\right)$ if and only if $\left(v, \varphi_{0}\right)=0$.

Let us now assume that $-\Delta+V$ has a simple zero energy resonance. This assumption is made in order not to complicate the analysis too much. It is also 
possible to carry out the analysis without this assumption [3] but the formulas obtained will be somewhat more complicated. With this assumption it follows from [3] that $k^{\prime}(\varepsilon)$ is analytic in $\varepsilon$ in a complex disc centered at zero with a possible branching point of finite order at $\varepsilon=0$, under the assumption that $V(x)$ is bounded with compact support. It is furthermore proved in [3] that $\alpha=k^{\prime}(0)$ for some resonance or eigenvalue of the form $\varepsilon k^{\prime}(\varepsilon)$ if and only if $\alpha$ is such that the following equation has a nontrivial solution

$$
\sum_{j=1}^{n}\left[\frac{\sqrt{-1} \alpha}{4 \pi} \delta_{i j}+\left(1-\delta_{i j}\right) G_{\alpha}\left(x_{i}-x_{j}\right)\right] c_{j}=0 .
$$

That is $\frac{i \alpha}{4 \pi}$ is the eigenvalue of the matrix $\left(1-\delta_{i j}\right) G_{\alpha}\left(x_{i}-x_{j}\right)$ or $\alpha$ solves the equation

$$
\operatorname{Det}\left|\frac{\sqrt{-1} \alpha}{4 \pi} \delta_{i j}+\left(1-\delta_{i j}\right) G_{\alpha}\left(x_{i}-x_{j}\right)\right|=0 \text {. }
$$

Moreover we have that if $\alpha$ is a simple solution of (5.9) then $k(\varepsilon)$ is analytic for small $\varepsilon$, and if $\alpha$ is a multiple solution, then $k(\varepsilon)$ may have a branching point at $\varepsilon=0$ of order at most the multiplicity of $\alpha$, and $k(\varepsilon)$ is a multiple valued analytic function in a complex neighborhood of $\varepsilon=0$. Hence we have the following

Theorem 4.1. Let $E(\varepsilon)=k(\varepsilon)^{2}$ be an eigenvalue or resonance of the multiple well problem $-\Delta+\sum_{i=1}^{n} V\left(x-\frac{1}{\varepsilon} x_{i}\right)$, where $-\Delta+V(x)$ has a zero energy resonance, and let us assume that $|k(\varepsilon)| \leqq c \varepsilon$. Then $k(\varepsilon)$ is analytic in a complex neighborhood of $\varepsilon=0$ with the only possible singularity a branching point of finite order at $\varepsilon=0$. Moreover the derivative $\alpha=k^{\prime}(0)$ at zero exists and is a solution of the equation

$$
\operatorname{Det}\left|\frac{\sqrt{-1} \alpha}{4 \pi} \delta_{i j}+\left(1-\delta_{i j}\right) G_{\alpha}\left(x_{i}-x_{j}\right)\right|=0,
$$

where $G_{\alpha}(x)=(4 \pi|x|)^{-1} e^{i \alpha|x|}$. If $\alpha$ is a simple solution, then $k(\varepsilon)$ is analytic near $\varepsilon=0$, and if $\alpha$ has multiplicity $\ell$, then $k(\varepsilon)$ has almost a branching point of order $\ell$ at zero. Conversely if $\alpha$ is a solution of the equation above, then there is a resonance or eigenvalue $E(\varepsilon)=k(\varepsilon)^{2}$, which is analytic for $\varepsilon$ in a complex neighborhood of $\varepsilon=0$ such that $k(0)=0$ and $k^{\prime}(0)=\alpha ; E(\varepsilon)$ is a resonance if $\operatorname{Im} \alpha \leqq 0$ and eigenvalue if $\operatorname{Im} \alpha>0$.

It is also proven in [3] that the solutions $\varphi_{i}(\varepsilon, x)$ of $(4.5)$ are analytic in $\varepsilon$ if $\alpha$ is a simple solution of (5.9) and that $\varphi_{j}(\varepsilon, x)=c_{j} \varphi_{0}(x)+\varepsilon \chi_{j}(\varepsilon, x)$, where $\chi_{j}(\varepsilon, x)$ is analytic. Here $\varepsilon$ and $c_{j}$ are the eigenvectors in (5.8). Since the corresponding eigenor resonance function is given by

$$
\psi_{\varepsilon}(x)=\sum_{i=1}^{n} \int G_{k(\varepsilon)}\left(x-y-\frac{1}{\varepsilon} x_{i}\right) v(y) \varphi_{i}(\varepsilon, y) d y .
$$

Using now that $\chi_{j}(\varepsilon, x)$ is uniformly bounded in $L_{2}$-norm and that $v(y)$ has compact support, we get using $\varphi_{j}=c_{j} \varphi^{0}+\varepsilon \chi_{j}$ and (5.7) that if $k(\varepsilon)^{2}$ is an eigenvalue then 


$$
\psi_{\varepsilon}(x)=\sum_{i=1}^{n} c_{j} \psi_{0}\left(x-\frac{1}{\varepsilon} x_{i}\right)+\varepsilon \chi_{\varepsilon}
$$

where $\left|\chi_{\varepsilon}(x)\right| \leqq c$ independent of $\varepsilon$ and $x$. Hence we get

Theorem 4.2. Let $E(\varepsilon)=k^{2}(\varepsilon)$ be an eigenvalue of the multiple well problem $-\Delta+\sum_{i=1}^{m} V\left(x-\frac{1}{\varepsilon} x_{i}\right)$, where $-\Delta+V(x)$ has a zero energy resonance, and let us assume that $|k(\varepsilon)| \leqq c \varepsilon$. Moreover let us assume that $\alpha=k^{\prime}(0)$ is a simple solution of

$$
\operatorname{Det}\left|\frac{\sqrt{-1} \alpha}{4 \pi} \delta_{i j}+\left(1-\delta_{i j}\right) G_{\alpha}\left(x_{i}-x_{j}\right)\right|=0 \text {. }
$$

If $\psi_{\varepsilon}(x)$ is the eigenfunction corresponding to $E(\varepsilon)$, then

$$
\psi_{\varepsilon}(x)=\sum_{i=1}^{n} c_{i} \psi_{0}\left(x-\frac{1}{\varepsilon} x_{i}\right)+\varepsilon \chi_{\varepsilon}(x),
$$

where $\left|\chi_{\varepsilon}(x)\right| \leqq c$ independent of $\varepsilon$ and $x$ and $\psi_{0}=G_{0} v \varphi_{0}$ is the zero energy resonance function of $-\Delta+V$ and $c_{i}$ satisfy

$$
\sum_{j}\left[\frac{1}{4 \pi} \sqrt{-1} \alpha \delta_{i j}+\left(1-\delta_{i j}\right) G_{\alpha}\left(x_{i}-x_{j}\right)\right] c_{j}=0 .
$$

\section{The Critical Double Well}

In this section we consider the Hamiltonian $H_{z}=-\Delta+V(x)+V(x-z)$, where $-\Delta+V$ has a zero energy resonance and $V$ is again bounded with compact support. From the previous section we then have that $H$ has a sequence of resonances $k_{n}^{ \pm}(z)^{2}$ which tend to zero as $z \rightarrow \infty$, such that

$$
k_{n}^{ \pm}(z)=\frac{\gamma_{n}^{ \pm}}{|z|}+O\left(|z|^{2}\right)
$$

where $\gamma_{n}^{ \pm}$are the complex solutions of

$$
i \gamma \pm e^{i \gamma}=0 .
$$

It is easy to see that (5.2) has exactly one solution in the upper half plane. This solution is $\gamma_{0}=i \sigma_{0}$, where $\sigma_{0}$ is the unique real solution of

$$
\sigma_{0}=e^{-\sigma_{0}} \text {. }
$$

Hence $H_{z}$ has only one eigenvalue tending to zero as $z \rightarrow \infty$ and

$$
E_{0}(z)=-\frac{\sigma_{0}^{2}}{|z|^{2}}+O\left(|z|^{-3}\right) .
$$

This eigenvalue $E_{0}(z)$ was found by Klaus and Simon in [1]. However in addition to the unique solution in the upper half-plane (5.2) has infinitely many solutions in the lower or unphysical half-plane. To see this we write $\gamma=x+i y$, and then (5.2) 
takes the form

$$
-y+i x= \pm e^{-y}(\cos x+i \sin x)
$$

or

$$
-\mathrm{ye}^{y}= \pm \cos x \quad \text { and } \quad x= \pm e^{-y} \sin x
$$

Hence

$$
e^{y}=\left|\frac{\sin x}{x}\right| \text { and } \mp \frac{\sin x}{x} \log \left|\frac{\sin x}{x}\right|= \pm \cos x,
$$

or simply

$$
\frac{\sin x}{x} \log \left(\left|\frac{\sin x}{x}\right|\right)=-\cos x
$$

Now $\frac{\sin x}{x}$ is zero at $n \pi$, so that the left hand side of $(5.7)$ is zero at $n \pi$, while the right hand side is zero at $\left(n+\frac{1}{2}\right) \pi$. It is easy to see that (5.7) has exactly one solution in each interval $\left(n \pi,\left(n+\frac{1}{2}\right) \pi\right)$. Hence we have the following theorem.

Theorem 5.1. Let $H_{z}=-\Delta+V(x)+V(x-z)$, where $-\Delta+V$ has a zero energy resonance and $V(x)$ is bounded with compact support. Then $H_{z}$ has exactly one eigenvalue $E_{0}(z)$ which tends to zero as $z \rightarrow \infty . E_{0}(z)$ is analytic in $\frac{1}{|z|}$ and

$$
E_{0}(z)=-\sigma_{0}^{2}|z|^{-2}+O\left(|z|^{-3}\right),
$$

where $\sigma_{0}$ is the unique real solution of $\sigma=e^{-\sigma}$. However $H_{z}$ has an infinite sequence of resonances $E_{n}(z), n=1,2, \ldots$, tending to zero as $z \rightarrow \infty$, such that

$$
E_{n}(z)=\gamma_{n}^{2}|z|^{-2}+O\left(|z|^{-3}\right),
$$

where $\gamma_{n}=x_{n}+i y_{n}, y_{n}<0$, where $x_{n}$ are the real solutions of

$$
\frac{\sin x}{x} \log \left(\left|\frac{\sin x}{x}\right|\right)=-\cos x
$$

and $y_{n}=\log \left(\left|\frac{\sin x}{x}\right|\right)$. There is exactly one solution in each interval $\left(n \pi,\left(n+\frac{1}{2}\right) \pi\right)$. Moreover for large $n$ we have

$$
x_{n} \sim\left(n+\frac{1}{2}\right) \pi, \quad y_{n} \sim-\log \left(\left(n+\frac{1}{2}\right) \pi\right),
$$

see Table 1. Table 2 gives the corresponding asymptotic resonances for three equal distant centers.

By utilizing the methods in [4] it is possible to prove that

$$
E_{n}(z)=\gamma_{n}^{2}|z|^{-2}+\left(\psi_{0}, V\right)^{-2}\left(\psi_{0}, V \psi_{0}\right) \frac{\left(i \gamma_{n}\right)^{3}}{1-i \gamma_{n}}|z|^{-3}+O\left(|z|^{-4}\right)
$$

where $\psi_{0}$ is the zero energy resonance function. 
Table 1. The asymptotic resonances for $H_{z}=-\Delta+V(x)$ $+V(x-z)$ as $|z| \rightarrow \infty$ are $E_{m}(z)$ $=\sigma_{n}^{2}|z|^{-2}+O\left(|z|^{-3}\right)$, where $\sigma_{n}$ $=\pi \cdot x_{n}+i y_{n}$ and the first few $x_{n}$ and $y_{n}$ are

\begin{tabular}{ll}
\hline$x_{n}$ & $y_{n}$ \\
\hline 0.425655 & -0.318132 \\
1.392665 & -1.533913 \\
2.415536 & -2.062278 \\
3.430203 & -2.401585 \\
4.440171 & -2.653192 \\
5.447408 & -2.853582 \\
6.452924 & -3.020240 \\
7.457284 & -3.162953 \\
8.460827 & -3.287769 \\
9.463770 & -3.398692 \\
10.466259 & -3.498515 \\
11.468394 & -3.589263 \\
12.470248 & -3.672450 \\
13.471876 & -3.749243 \\
14.473317 & -3.820554 \\
15.474603 & -3.887116 \\
16.475759 & -3.949523 \\
17.476803 & -4.008262 \\
18.477753 & -4.063742 \\
19.478621 & -4.116305 \\
20.479416 & -4.166242 \\
&
\end{tabular}

Table 2. The asymptotic resonances for $H_{\varepsilon}=-\Delta+\sum_{\imath=1}^{3}$ $\cdot V\left(x-\frac{1}{\varepsilon} x_{i}\right)$ as $\varepsilon \rightarrow 0$, where

$\left|x_{1}-x_{2}\right|=\left|x_{2}-x_{3}\right|=\left|x_{1}-x_{3}\right|=$ 1

are $E_{n}(\varepsilon)=\sigma_{n}^{2} \varepsilon^{2}+O\left(\varepsilon^{2}\right)$, where $\sigma_{n}=\pi \cdot x_{n}+i y_{n}$, and the first few $x_{n}$ and $y_{n}$ are

\begin{tabular}{ll}
\hline$x_{n}$ & $y_{n}$ \\
\hline 0.425655 & -0.318132 \\
1.442028 & -0.834310 \\
2.415536 & -2.062278 \\
3.450418 & -1.702259 \\
4.440171 & -2.653192 \\
5.460185 & -2.156909 \\
6.452924 & -3.020240 \\
7.466639 & -2.467530 \\
8.460827 & -3.287769 \\
9.471153 & -2.703946 \\
10.466259 & -3.498515 \\
11.474492 & -2.894923 \\
12.470248 & -3.672450 \\
13.477071 & -3.055169 \\
14.473317 & -3.820554 \\
15.479128 & -3.193227 \\
16.475759 & -3.949523 \\
17.480812 & -3.314505 \\
18.477753 & -4.063742 \\
19.482218 & -3.422646 \\
20.479416 & -4.166242 \\
\hline &
\end{tabular}

Acknowledgements. It is a pleasure to thank Professor G. Jona-Lasinio, Dr. F. Gesztesy, and Professor $\mathrm{S}$. Albeverio for helpful discussions concerning the problems in connection with this paper.

\section{References}

1. Klaus, M., Simon, B.: Binding of Schrödinger particles through conspiracy of potential Wells. Ann. Inst. Henri Poincaré 30, 83-87 (1979)

2. Klaus, M.: Some remarks on Double-Wells in one and three dimensions. Ann. Inst. Henri Poincaré 30, 405-417 (1981)

3. Holden, H., Høegh-Krohn, R., Johannesen, S.: The short range expansion, Adv. Appl. Math. (to appear)

4. Holden, H., Høegh-Krohn, R., Johannesen, S.: The short range expansion in solid state. Preprint Math. Inst. Oslo Univ.

Communicated by B. Simon

Received June 14, 1982; in revised form April 14, 1983 
